\title{
FOXL2 molecular status in adult granulosa cell tumors of the ovary: A study of primary and metastatic cases
}

\author{
GIAN FRANCO ZANNONI ${ }^{1}$, GIUSEPPINA IMPROTA ${ }^{2}$, MARCO PETRILLO ${ }^{3}$, \\ ANGELA PETTINATO $^{4}$, GIOVANNI SCAMBIA ${ }^{3}$ and FILIPPO FRAGGETTA ${ }^{4}$ \\ ${ }^{1}$ Department of Pathology, Catholic University of The Sacred Heart, I-00168 Rome; \\ ${ }^{2}$ Laboratory of Clinical Research and Advanced Diagnostics, Hospitalization and Treatment Institute Scientific- \\ Oncological Referral Center of Basilicata, I-85028 Potenza; ${ }^{3}$ Department of Obstetrics and Gynecology, \\ Catholic University of the Sacred Heart, I-00168 Rome; ${ }^{4}$ Department of Pathology, Cannizzaro Hospital, I-95126 Catania, Italy
}

Received June 4, 2015; Accepted April 29, 2016

DOI: $10.3892 / 01.2016 .4711$

\begin{abstract}
Granulosa cell tumors (GCTs) of the ovary are uncommon neoplasms, accounting for $\sim 5 \%$ of all malignant ovarian tumors. GCTs are a relatively homogeneous group of tumors, categorized into two distinct subtypes, juvenile GCT and adult GCT (AGCT), likely arising from a limited set of molecular events usually involving the disruption of pathways that regulate granulosa cell proliferation. In the present study, the presence of forkheadbox L2 (FOXL2) c.402C $>$ G mutation was investigated in a series of 42 samples of primary and metastatic AGCT of the ovary. The samples consisted of 37 primary and 5 metastatic ovarian AGCTs from 37 patients. FOXL2 mutational status was evaluated using a pyrosequencing approach on $2.5-\mu \mathrm{m}$ sections of formalin-fixed paraffin-embedded tissue. FOXL2 c.402C $>$ G mutation was found in 33/37 (89.2\%) primary AGCTs and in 4/5 (80.0\%) metastases, with the molecular status of the metastases recapitulating that of the primary tumors (4 mutated cases and 1 wild-type case). Overall, FOXL2 mutation is present in the majority of primary and metastatic AGCTs, and could be used as a valid tool in the diagnosis of the disease and in cases of metastatic lesions from an unknown primary origin. Moreover the concordance of FOXL2 molecular status in primary and associated metastases suggests its early appearance and genomic stability in AGCT tumorigenesis.
\end{abstract}

Correspondence to: Dr Giuseppina Improta, Laboratory of Clinical Research and Advanced Diagnostics, Hospitalization and Treatment Institute Scientific-Oncological Referral Center of Basilicata, Via Padre Pio 1, Rionero in Vulture, I-85028 Potenza, Italy

E-mail: giuseppina.improta@gmail.com

Key words: FOXL2, granulosa cell tumors, ovary, mutation, molecular signatures

\section{Introduction}

Granulosa cell tumors (GCTs) of the ovary are uncommon neoplasms, accounting for $~ 5 \%$ of all malignant ovarian tumors (1). The tumors can be further categorized into two distinct subtypes, juvenile GCT (JGCT) and adult GCT (AGCT), with the latter accounting for $95 \%$ of GCTs and most commonly presenting during the perimenopausal or early postmenopausal period (2).

AGCTs are characterized by slow, indolent growth, with a favorable prognosis and a tendency to late recurrence; however, those patients who experience recurrence and/or are at an advanced stage, have a poor prognosis, with $\sim 80 \%$ of them succumbing to the disease (2). The majority of recurrences occur in the peritoneal cavity, although the retroperitoneal lymph nodes, upper abdominal solid organs, lungs and skeleton may be involved (3).

GCTs are considered to be a relatively homogeneous group of tumors, likely arising from a limited set of molecular events, usually involving the disruption of pathways that regulate granulosa cell proliferation $(4,5)$. Indeed, GCTs exhibit a gene expression profile consistent with that of normal proliferating granulosa cells of preantral follicles (6-8).

In 2009, Shah et al (9) identified a single somatic missense mutation (p.C134W) in the forkheadbox L2 (FOXL2) gene, which was present in $97 \%$ of AGCTs and absent in other tumors, suggesting it was of diagnostic significance, as demonstrated by other two different studies (9-11).

In the present study, the molecular status of FOXL2 was analyzed in a series of 37 primary ovarian AGCTs and 5 associated metastases.

\section{Patients and methods}

Case selection. A total of 37 consecutive patients with a proven diagnosis of AGCT of the ovary were included in the present study. Metastases developed in 5 of these patients, therefore, a final series of 42 samples (37 primary and 5 metastases) were available for the study. In all cases, the histological diagnosis was formulated after an extensive and careful evaluation of tumor specimens by an experienced gynecological pathologist. 


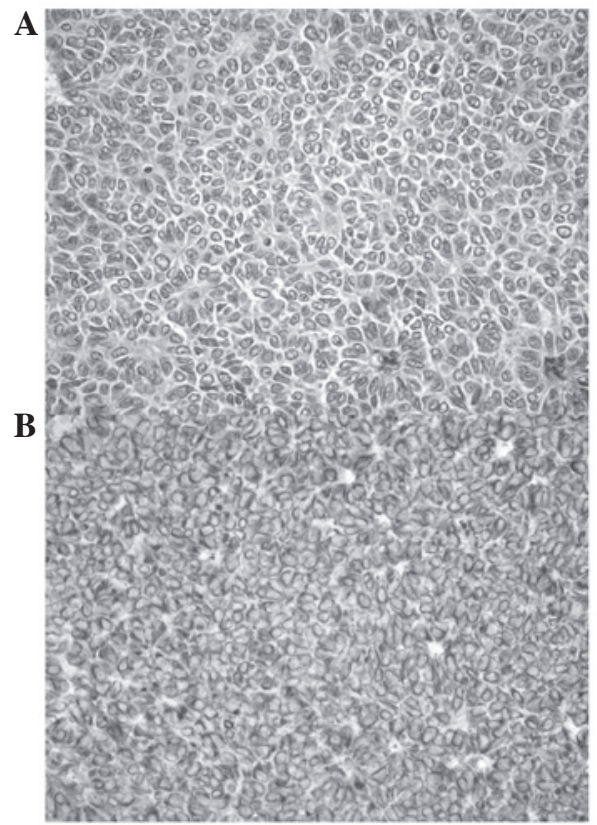

Figure 1. Ovarian adult granulosa cell tumor (A) showing increased mitotic activity and (B) a high level of $\alpha$-inhibin expression by immunohistochemical analysis (magnification, x100).

In all cases, according to International Federation of Gynecology and Obstetrics (FIGO) and World Health Organization (WHO) criteria $(12,13)$, a final diagnosis of AGCT of the ovary was formulated. At the time of admission to hospital, patients provided written informed consent for their clinical data to be collected and analyzed for research purposes. All tissue samples were obtained from the Catholic University of The Sacred Heart (Rome, Italy). The study was approved the institutional review board of the Catholic University of The Sacred Heart.

Molecular analysis. The PyroMark Q24TM system (Qiagen $\mathrm{GmbH}$, Hilden, Germany) was used for the pyrosequencing analysis of FOXL2, exon 1, codon 134 mutations, from formalin fixed paraffin-embedded tissues of primary GCTs and associated metastases. Briefly, all the slides were reviewed by a pathologist to evaluate the percentage of tumor cells (>90\% tumor cells) prior to performing the manual dissection of the tumor area, thus avoiding the samples containing tumor necrosis or few neoplastic cells. Next, all tumors underwent genomic DNA extraction, using the QIAamp DNA FFPE Tissue kit (Qiagen $\mathrm{GmbH}$ ), according to the manufacturer's protocols. A custom Pyro assay was designed and a 50-bp region containing codon 134 of FOXL2 was amplified by polymerase chain reaction (PCR; GeneAmp PCR System 9700; Applied Biosystems Inc., Foster City, CA, USA) using biotinylated forward (5'-CAACTACTGGACGCTGGA CC-3') and unlabeled reverse (5'-TGCCCTTCTCGAACA TGTCT-3') primers. The cycling conditions were as follows: Initial step at $95^{\circ} \mathrm{C}$ for $15 \mathrm{~min}$, followed by 45 cycles of denaturation at $94^{\circ} \mathrm{C}$ for $30 \mathrm{sec}$, annealing at $60^{\circ} \mathrm{C}$ for $30 \mathrm{sec}$ and extension at $72^{\circ} \mathrm{C}$ for $30 \mathrm{sec}$, and a final extension at $72^{\circ} \mathrm{C}$ for $10 \mathrm{~min}$. A unidirectional primer (5'-CCTTCTCGAACA TGTCT-3') targeting the coding strand was used to detect the c.402C $>$ G, p.Cys134Trp mutation by pyrosequencing

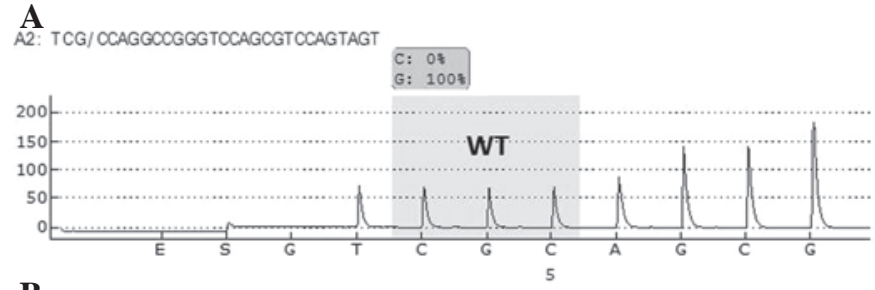

B

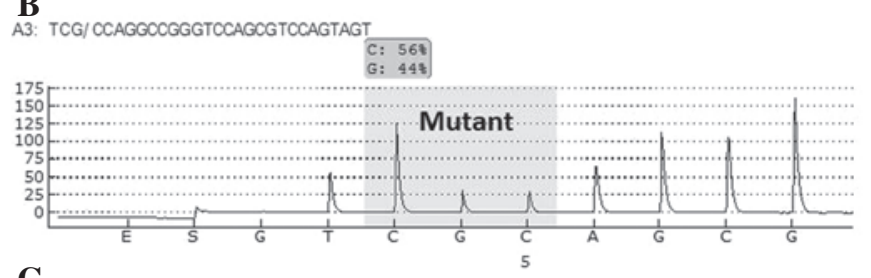

A1: TCG/ OCAGGOOGGGTOCAGCGTOCAGTAGT

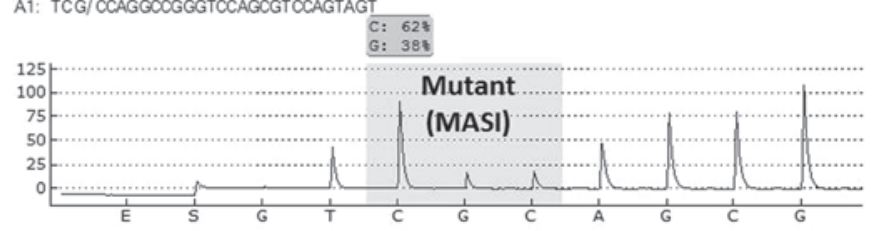

Figure 2. (A) Pyrogram trace of an AGCT wild-type case showing the absence of FOXL2 p.C134W mutation. (B) Pyrogram trace of an AGCT mutant case showing the presence of FOXL2 p.C134W mutation with a mutant allele population of $\sim 56 \%$; (C) Pyrogram trace of an AGCT mutant case showing the presence of FOXL2 p.C134W mutation with a mutant allele-specific imbalance of $\sim 62 \%$ (MASI). AGCT, adult granulosa cell tumor; MASI, mutant allele-specific imbalance; WT, wild-type; FOXL2, forkheadbox L2.

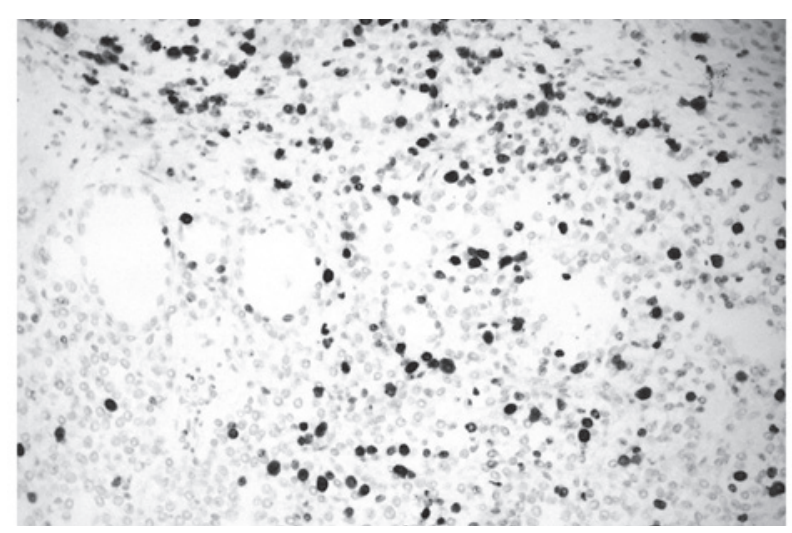

Figure 3. Ovarian adult granulosa cell tumor showing a high level of Ki-67 expression (immunohistochemical staining; magnification, x100).

using the PyroMark Q24TM system (Qiagen $\mathrm{GmbH}$ ), and the PyroMark TM-Q24 software (Qiagen $\mathrm{GmbH}$ ) was used for data analysis.

\section{Results}

The median age of the study population was 46 years (range, 24-75), with 5/37 (13.5\%) women at FIGO stage II, in line with literature data (14-16). According to the WHO classification of tumors of the female reproductive organs (13), histological features, including uniform pale and round nuclei and nucleargrooves, and $\alpha$-inhibin immunohistochemistry were consistent with the diagnosis of AGCT in all cases (Fig. 1). All samples tested for the mutation contained at least $90 \%$ tumor cells by histological evaluation. In total, 33 out of $37(89.2 \%)$ 
Table I. Percentage of FOXL2 c.402G $>$ C mutation in AGCTs shown in the literature.

\begin{tabular}{lcc}
\hline First author, year & $\begin{array}{c}\text { \% FOXL2 } \\
\text { AGCT mutation }\end{array}$ & (Ref.) \\
\hline Shah et al, 2009 & 97.0 & $(9)$ \\
Schrader et al, 2009 & 97.0 & $(10)$ \\
Kim et al, 2010 & 94.6 & $(11)$ \\
Rosario et al, 2013 & 69.2 & $(14)$ \\
D'Angelo et al, 2011 & 70.0 & $(15)$ \\
Kommoss et al, 2014 & 94.0 & $(16)$ \\
Kim et al, 2010 & 97.0 & $(25)$ \\
Jamieson et al, 2010 & 93.0 & $(26)$ \\
Al-Agha et al, 2011 & 93.0 & $(27)$ \\
Benayoun et al, 2010 & 95.0 & $(28)$ \\
Present study & 89.0 & \\
\hline
\end{tabular}

FOXL2, forkheadbox L2; AGCT, AGCT, adult granulosa cell tumor.
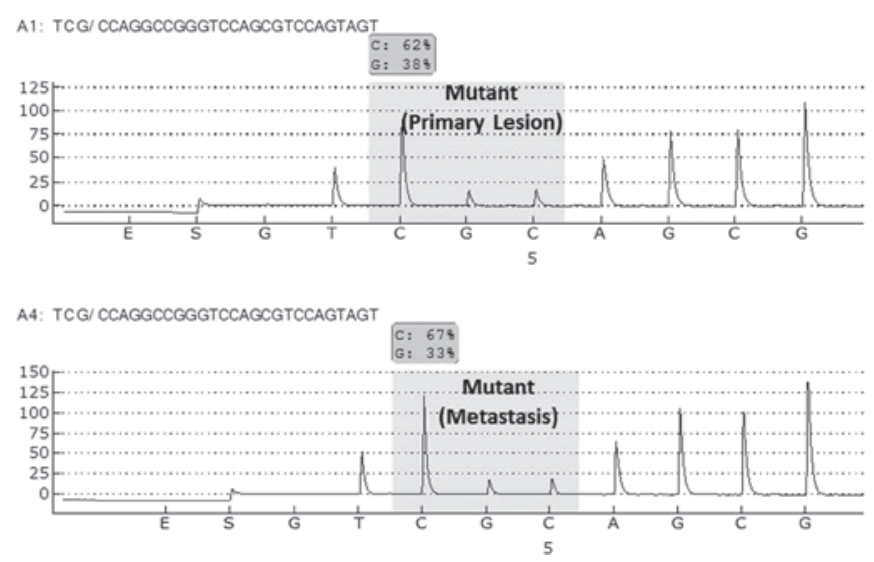

Figure 4. Pyrogram traces of a primary adult granulosa cell tumor mutan case and its associated metastasis showing the full concordance and the stability of forkheadbox L2 p.C134W mutation.

primary AGCTs exhibited the FOXL2 c.402C $>$ G mutation on pyrosequencing analysis. Only 6/33 (18.2\%) presented with a wild-type molecular profile (Fig. 2A); the majority of mutated tumors $(27 / 33 ; 81.8 \%)$ showed a heterozygous pattern with a mutant allele population of $\sim 50 \%$ (Fig. 2B), whereas 6/33 mutated samples (18.2\%) showed a non-heterozygous pattern with a mutant allele-specific imbalance (MASI) of $\sim 70 \%$ (Fig. 2C).

On the basis of the molecular status, the 4 cases showing no FOXL2 c. $402 \mathrm{C}>\mathrm{G}$ mutation (wild-type cases) were reviewed, and the diagnoses of AGCT were confirmed on the basis of morphological and immunohistochemical findings. However, all the primary ovarian wild-type cases (4/4) presented with a high mitotic index, as shown by Ki-67 expression (Fig. 3).

With regard to the metastases, $4 / 5(80.0 \%)$ cases presented with the FOXL2 c. $402 \mathrm{C}>\mathrm{G}$ mutation, with full concordance between the FOXL2 c.402G $>\mathrm{C}$ mutation in primary and matched metastases. Notably, in one case, the MASI was found in the ovarian primary tumor and the associated metastasis
(Fig. 4). The other metastatic samples (as well as their associated primaries) showed no MASI.

\section{Discussion}

The FOXL2 gene is located on chromosome band $3 \mathrm{q} 22.3$ and encodes forkhead box protein L2, a forkhead-winged helix family of transcription factors, whose expression has been consistently observed in the developing eyelids and predominantly in the granulosa cells of fetal and adult ovaries (17-20). In the ovary, FOXL2 plays an important role in the formation of follicles and is required for ovarian maintenance $(17,21)$.

FOXL2 is involved in multiple dysfunctional states in the human ovary. Germline mutations in FOXL2 lead to the development of blepharophimosis, ptosis and epicanthus inversus syndrome, with or without accompanying premature ovarian failure (POF) (22). In addition, FOXL2 has also been implicated in sporadic POF $(23,24)$. Indeed, somatic mutations located in the forkhead domain of FOXL2 have been associated with the development of AGCT. In 2009, Shah et al (9) used whole transcriptome 'next-generation' sequencing on 4 AGCT cases and first identified a unique recurring somatic missense mutation, p.C134W (c.402C $>$ G) in the FOXL2 gene. Subsequently, using direct sequencing, this mutation was confirmed in $97 \%$ of a cohort of 89 patients affected by AGCTs and in only 1 out of the 10 JGCTs (9). Notably, other studies on a variety of tumors have demonstrated the high specificity of the p.C134W mutation for ovarian AGCT, with the FOXL2 p.C134W mutation found in $>90 \%$ of AGCTs and $10-20 \%$ of ovarian thecomas in multiple case series $(9,25-27)$. The incidence of the FOXL2 p.C134W mutation in the present study was $89 \%$, a result in line with certain literature data, but slightly lower than other reported incidences of $94-97 \%$ for mutant adult-type tumors (Table I) (9-11,14-16,26,28).

The variation in the reported incidence, ranging from $70-100 \%$ may be associated with different causes, such as the sensibility of the methodologies used in detecting the mutation, and in the case and patient selection (i.e., different geographical areas with different incidences). In particular, Kommoss et al (16) reported an initial FOXL2 mutation rate of $87 \%$ using Sanger sequencing analysis, and then a rate of $94 \%$ using a Taq-Man assay approach of higher sensitivity. Moreover, in this study, following expert review, Kommoss et al confirmed the diagnosis of AGCT in only 2 of the 6 initial wild-type AGCTs: 1 case was classified as a pure wild-type AGCT and another case was diagnosed as a mixed adult and juvenile wild-type GCT (16).In the present series, the FOXL2 wild-type cases were reviewed and the initial diagnosis of AGCT was confirmed in all cases, on the basis of the morphological and immunohistochemical findings. However, all these cases presented with an elevated mitotic index, as shown by $\mathrm{Ki}-67$ expression. On the basis of these findings, certain considerations should be taken into account: If the hot-spot FOXL2 mutation p.C134W is considered specific to AGCT, one could argue that the reported wild-type cases simply represent tumors resembling AGCT instead of true AGCT, on the basis of the morphological and immunohistochemical findings. Larger series, with clinical findings and follow-up data, are required to support this hypothesis and to identify wild-type AGCT as a distinct clinicopathological entity. 
Little is known regarding relapsing or metastasizing AGCTs and FOXL2 molecular status, and to the best of our knowledge, this is the first series (albeit small) on the topic. Among 5 cases of ovarian primary AGCT and their associated metastases, the present data showed 4 cases of FOXL2 c. $402 \mathrm{G}>\mathrm{C}$ mutation ( $80 \%$ ) and 1 wild-type case (20\%), demonstrating the total concordance of the AGCT molecular status and highlighting the early appearance of p.C134W mutation in AGCT along with its genomic stability (Fig. 3). This data is in keeping with the 2013 study by Kommoss et al (29), which highlighted the utility of FOXL2 mutation testing in the original and relapsed tumor, in a single case of unclear or mistaken gynecological diagnosis (29). As a practical consequence, in cases of metastasis from unknown primary lesions, the positivity of the molecular analysis of FOXL2 could assist the pathologist in finding the correct diagnosis of GCT of the ovary, as shown by the present data.

According to the present study findings, the FOXL2 p.C134W mutation could be considered as an almost universal feature of AGCT, supporting the application of molecular analysis for FOXL2 to differentiate this tumor from other ovarian malignancies $(9,11)$. Moreover, in the present study, the concordance of the FOXL2 molecular status in primary and metastatic AGCT was shown, suggesting this mutation to be an early event in AGCT tumorigenesis.

To date, few studies have investigated the pathogenicity and the clinical significance of the FOXL2 p.C134W mutation. The $402 \mathrm{C}>\mathrm{G}$ mutation results in a substitution of cysteine with tryptophan at amino acid position 134 (Cys134Trp). Computer modeling suggests that the presence of tryptophan does not disrupt the folding of the forkhead domain or its ability to interact with DNA, but that it may affect the interaction with other transcription factors (9). Actually, previous unbiased whole transcriptome analyses of GCT patient samples and cell lines indicated that FOXL2 with p.C134W mutation is unable to downregulate genes involved in the control of the cell cycle, and does not upregulate (as wild-type FOXL2) genes involved in cell death $(30,31)$.

The clinical significance of FOXL2 and the type of its mutation (i.e., MASI or not) is a moot point. The present study reported 6 cases of AGCT showing FOXL2 mutations of MASI type. MASI has been previously reported in several tumors harboring oncogenic mutations, which are most commonly due to copy number gain or copy neutral loss of heterozygosity $(32,33)$. MASI has been shown to impact the mutant allele transcription levels, and increased oncogene function has been noted in tumors with an excess mutant allele population. MASI has therefore been implicated as a possible tumor progression factor (32). MASI for FOXL2 p.C134W mutation has rarely been reported in cases of AGCT. According to Rosario et al (14), tumors showing MASI have to be homozygous for FOXL2 mutation rather than hemizygous; this conclusion is in contrast to that of previously published literature data $(33,34)$. Notably, patients believed to be homozygous for p.C134W mutation have demonstrated significantly higher relapse rates, and the mutant tumors tended to exhibit higher FOXL2 expression than wild-type tumors, suggesting that the mutant FOXL2 allele may increase the expression of mutant FOXL2, possibly with involvement in recurrent disease $(14,15)$. This, however, is not supported by the present data, since only $1 / 5$ metastatic AGCTs was associated with a MASI status of FOXL2 mutation, thus suggesting that other studies are required to better understand the clinical significance of the FOXL2 mutation in AGCT.

In conclusion, the present study showed that AGCT of the ovary typically presents with FOXL2 mutation, and that this mutation is maintained even in metastatic AGCT, suggesting that this is an early event in AGCT pathogenesis. The results of the present study suggest the use of the molecular study of FOXL2 as a diagnostic tool in primary AGCT and its metastases.

\section{References}

1. Colombo N, Parma G, Zanagnolo V and Insinga A: Management of ovarian stromal cell tumors. J Clin Oncol 25: 2944-2951, 2007.

2. Young RH and Scully RE: Endocrine tumors of the ovary. Curr Top Pathol 85: 113-164, 1992.

3. Fox H, Agrawal K and Langley FA: A clinicopathologic study of 92 cases of granulosa cell tumor of the ovary with special reference to the factors influencing prognosis. Cancer 35: 231-241, 1975.

4. Fuller PJ, Chu S, Fikret S and Burger HG: Molecular pathogenesis of granulosa cell tumours. Mol Cell Endocrinol 191: 89-96, 2002.

5. Jamieson S and Fuller PJ: Management of granulosa cell tumour of the ovary. Curr Opin Oncol 20: 560-564, 2008

6. Chu S, Rushdi S, Zumpe ET, Mamers P, Healy DL, Jobling T, Burger HG and Fuller PJ: FSH-regulated gene expression profiles in ovarian tumours and normal ovaries. Mol Hum Reprod 8: 426-433, 2002.

7. Fuller PJ and Chu S: Signaling pathways in the molecular pathogenesis of ovarian granulosa cell tumours. Trends Endocrinol Metab 15: 122-128, 2004.

8. Anttonen M, Unkila-Kallio L, Leminen A, Butzow R and Heikinheimo M: High GATA-4 expression associates with aggressive behavior, whereas low anti-Müllerian hormone expression associates with growth potential of ovarian granulosa cell tumors. J Clin Endocrinol Metab 90: 6529-6235, 2005.

9. Shah SP, Köbel M, Senz J, Morin RD, Clarke BA, Wiegand KC, Leung G, Zayed A, Mehl E, Kalloger SE, et al: Mutation of FOXL2 in granulosa-cell tumors of the ovary. N Engl J Med 360: 2719-2729, 2009.

10. Schrader KA, Gorbatcheva B, Senz J, Heravi-Moussavi A, Melnyk N, Salamanca C, Maines-Bandiera S, Cooke SL, Leung P, Brenton JD, et al: The specificity of the FOXL2 c.402C $>$ G somatic mutation: A survey of solid tumors. PLoS One 4: e7988, 2009.

11. Kim MS, Hur SY, Yoo NJ and Lee SH: Mutational analysis of FOXL2 codon 134 in granulosa cell tumour of ovary and other human cancers. J Pathol 221: 147-152, 2010.

12. Berek JS, Crum C and Friedlander M: Cancer of the ovary, fallopian tube, and peritoneum. Int J Gynaecol Obstet 131 (Suppl 2): S111-S122, 2015.

13. Zaloudek CJ, Mooney EE, Staats PN and Young RH: Tumours of the ovary and peritoneum. In: World Health Organization Classification of Tumours: Pathology and Genetics of Tumours of the Breast and Female Genital Organs. Kurman R, Carcangiu ML, Herrington CS and RH Young (eds). IARC Press, Lyon, p146, 2014.

14. Rosario R, Wilson M, Cheng WT, Payne K, Cohen PA, Fong P and Shelling AN: Adult granulosa cell tumours (GCT): Clinicopathological outcomes including FOXL2 mutational status and expression. Gynecol Oncol 131: 325-329, 2013.

15. D’Angelo E, Mozos A, Nakayama D, Espinosa I, Catasus L, Muñoz J and Prat J: Prognostic significance of FOXL2 mutation and mRNA expression in adult and juvenile granulosa cell tumors of the ovary. Mod Pathol 24: 1360-1367, 2011.

16. Kommoss S, Gilks CB, Penzel R, Herpel E, Mackenzie R, Huntsman D, Schirmacher P, Anglesio M, Schmidt D and Kommoss F: A current perspective on the pathological assessment of FOXL2 in adult-type granulosa cell tumours of the ovary. Histopathology 64: 380-388, 2014.

17. Uhlenhaut NH, Jakob S, Anlag K, Eisenberger T, Sekido R, Kress J, Treier AC, Klugmann C, Klasen C, Holter NI, et al: Somatic sex reprogramming of adult ovaries to testes by FOXL2 ablation. Cell 139: 1130-1142, 2009. 
18. Cocquet J, De Baere E, Gareil M, Pannetier M, Xia X, Fellous M and Veitia RA: Structure, evolution and expression of the FOXL2 transcription unit. Cytogenet Genome Res 101: 206-211, 2003.

19. Cocquet J, Pailhoux E, Jaubert F, Servel N, Xia X, Pannetier M, De Baere E, Messiaen L, Cotinot C, Fellous M and Veitia RA: Evolution and expression of FOXL2. J Med Genet 39: 916-921, 2002.

20. Crisponi L, Deiana M, Loi A, Chiappe F, Uda M, Amati P, Bisceglia L, Zelante L, Nagaraja R, Porcu S, et al: The putative forkhead transcription factor FOXL2 is mutated in blepharophimosis/ptosis/epicanthus inversus syndrome. Nat Genet 27: 159-166, 2001.

21. Duffin K, Bayne RA, Childs AJ, Collins C and Anderson RA: The forkhead transcription factor FOXL2 is expressed in somatic cells of the human ovary prior to follicle formation. Mol Hum Reprod 15: 771-777, 2009.

22. Crisponi L, Uda M, Deiana M, Loi A, Nagaraja R, Chiappe F, Schlessinger D, Cao A and Pilia G: FOXL2 inactivation by a translocation $171 \mathrm{~kb}$ away: Analysis of $500 \mathrm{~kb}$ of chromosome 3 for candidate long-range regulatory sequences. Genomics 83 . 757-764, 2004.

23. Beysen D, Vandesompele J, Messiaen L, De Paepe A and De Baere E: The human FOXL2 mutation database. Hum Mutat 24: 189-193, 2004

24. Harris SE, Chand AL, Winship IM, Gersak K, Aittomäki K and Shelling AN: Identification of novel mutations in FOXL2 associated with premature ovarian failure. Mol Hum Reprod 8: 729-733, 2002

25. Kim T, Sung CO, Song SY, Bae DS and Choi YL: FOXL2 mutation in granulosa-cell tumours of the ovary. Histopathology 56: 408-410, 2010

26. Jamieson S, Butzow $R$, Andersson N, Alexiadis $M$ Unkila-Kallio L, Heikinheimo M, Fuller PJ and Anttonen M: The FOXL2 C134 W mutation is characteristic of adult granulosa cell tumors of the ovary. Mod Pathol 23: 1477-1485, 2010 .
27. Al-Agha OM, Huwait HF, Chow C, Yang W, Senz J, Kalloger SE, Huntsman DG, Young RH and Gilks CB: FOXL2 is a sensitive and specific marker for sex cord-stromal tumors of the ovary. Am J Surg Pathol 35: 484-494, 2011.

28. Benayoun BA, Caburet S, Dipietromaria A, Georges A, D'Haene B, Pandaranayaka PJ, L'Hôte D, Todeschini AL, Krishnaswamy S, Fellous M, et al: Functional exploration of the adult ovarian granulosa cell tumor-associated somatic FOXL2 mutation p.Cys134Trp (c.402C>G). PLoS One 5: e8789, 2010.

29. Kommoss S, Anglesio MS, Mackenzie R, Yang W, Senz J, Ho J, Bell L, Lee S, Lorette J, Huntsman DG and Blake Gilks C: FOXL2 molecular testing in ovarian neoplasms: Diagnostic approach and procedural guidelines. Mod Pathol 26: 860-867, 2013.

30. Benayoun BA, Anttonen M, L'Hôte D, Bailly-Bechet M, Andersson N, Heikinheimo M and Veitia RA: Adult ovarian granulosa cell tumor transcriptomics: Prevalence of FOXL2 target genes misregulation gives insights into the pathogenic mechanism of the p.Cys134Trp somatic mutation. Oncogene 32: 2739-2746, 2013.

31. Rosario R, Araki H, Print CG and Shelling AN: The transcriptional targets of mutant FOXL2 in granulosa cell tumours. PLoS One 7: e46270, 2012.

32. Soh J, Okumura N, Lockwood WW, Yamamoto H, Shigematsu H, Zhang W, Chari R, Shames DS, Tang X, MacAulay C, et al: Oncogene mutations, copy number gains and mutant allele specific imbalance (MASI) frequently occur together in tumor cells. PLoS One 4: e7464, 2009.

33. Improta G, Pelosi G, Tamborini E, Donia M, Santinami M, de Braud F and Fraggetta F: Biological insights into BRAFV600 mutations in melanoma patient: Not mere therapeutic targets. Oncoimmunology 2: e25594, 2013.

34. Geiersbach KB, Jarboe EA, Jahromi MS, Baker CL, Paxton CN, Tripp SR and Schiffman JD: FOXL2 mutation and large-scale genomic imbalances in adult granulosa cell tumors of the ovary. Cancer Genet 204: 596-602, 2011 